RACHEL BONNEMA, MD, MS Associate Professor of Medicine, Division of General Internal Medicine, University of Nebraska Medical Center, Omaha
MEGAN McNAMARA, MD, MSc Associate Professor of Medicine, Case Western Reserve University School of Medicine, Cleveland $\mathrm{OH}$; Louis Stokes Cleveland VA Medical Center
JENNIFER HARSH, PhD, LMFT Assistant Professor, Division of General Internal Medicine, University of Nebraska Medical Center, Omaha
ELIZABETH HOPKINS, PT, DPT The Nebraska Medical Center, Omaha

\title{
Primary care management of chronic pelvic pain in women
}

\section{ABSTRACT}

Chronic pelvic pain in women can arise from many causes and often results in significant declines in function and quality of life. A systematic approach for evaluating patients and initiating a management plan are recommended in the primary care setting. Comprehensive management strategies may include medication, pelvic physical therapy, and behavioral interventions.

\section{KEY POINTS}

Diagnosing and managing chronic pelvic pain may be difficult, but patients are often best served when their primary care provider directs a team-based approach to their care.

A detailed history, thorough abdominal and pelvic examinations, and targeted testing facilitate the diagnosis.

As in other chronic pain syndromes, the goals of therapy should be incremental and meaningful improvements in pain, function, and overall well-being.
C HRONIC PELVIC PAIN is a common clinical problem in women, as prevalent in primary care as asthma or back pain., ${ }^{1,2}$ It is often associated with lost work days and decreased productivity, increased healthcare spending, mood disorders, and negative effects on personal relationships. ${ }^{1-3}$

While specialty care referral may eventually be indicated, primary care doctors can take steps to diagnose and effectively manage the condition.

\section{COMPREHENSIVE MANAGEMENT LED BY PRIMARY CARE}

Chronic pelvic pain is defined as pain in the lower abdomen persisting for 3 to 6 months and of sufficient severity to require medical care or cause a functional disability. ${ }^{3}$ It is often detrimental to a woman's personal life and overall health, making a comprehensive assessment and multidisciplinary approach to management especially important.

The ideal care-delivery model is the patientcentered medical home, whereby a primary care physician coordinates comprehensive care with the help of an interdisciplinary team. 4.5 For complex cases, referral may be needed to other specialties (eg, obstetrics and gynecology, pain medicine) to help manage care.

\section{TARGETED EVALUATION}

Chronic pelvic pain often coexists with other systemic pain syndromes or psychiatric conditions common in primary care. Table 1 lists common causes and associated findings.

\section{Detailed history is critical}

The history is of utmost importance. Clinicians should query patients about the char- 


\section{TABLE 1}

\section{Common causes of chronic pelvic pain and associated findings}

Differential diagnosis Associated historical feature Physical examination

\section{Musculoskeletal}

Pelvic floor dysfunction

Complicated delivery, dyspareunia

Vaginismus, point tenderness, or high tone of pelvic floor

Myofascial pain or fibromyalgia Tender points, chronic somatic pain

Tender points

Stress fractures

Pain with repetitive movements, improved with rest

Degenerative disk disease

Burning, paresthesias

Radicular signs, muscle weakness

\section{Gastrointestinal}

Constipation

Inflammatory bowel disease

Bowel urgency, hematochezia

Irritable bowel syndrome

Bowel symptoms; may increase premenstrually

\section{Gynecologic (often cyclic with menses)}

Adhesions

Adenomyosis

Adnexal mass

Chronic pelvic inflammatory

disease

Dysmenorrhea

Endometrial or cervical polyp

Endometritis

Endometriosis

Leiomyomata

Pelvic congestion syndrome

Vulvar vestibulitis
Surgical history

Menorrhagia, dysmenorrhea

Localized to 1 area

Uterine cramping with menses

Intermenstrual or postcoital bleeding

Diffuse pelvic pain with menses, deep dyspareunia

Menorrhagia, pressure or heaviness

Multigravid patient; deep dyspareunia, postcoital pain, worse after prolonged standing

Vulvodynia, dyspareunia
Immobile uterus, nodularity

Enlarged, irregular tender uterus on bimanual examination

Localized adnexal mass

\section{Urologic}

Interstitial cystitis

Urgency, increased frequency of urination

Urinary tract infection

Dysuria

Urolithiasis

Localized sharp pain

Radiation cystitis

History of radiation

\section{Other}

Psychiatric (depression, somatization)

Neurologic (herpes zoster, nerve entrapment)
Concurrent mood disorder

Hot, burning, electric shock-like pain; shingles
Visual inspection

Uterine tenderness on bimanual examination

Fixed or immobile uterus, nodularity

Uterine nodularity, enlargement

Varicosity of labia, uterine tenderness on bimanual examination

Exquisite localized tenderness 


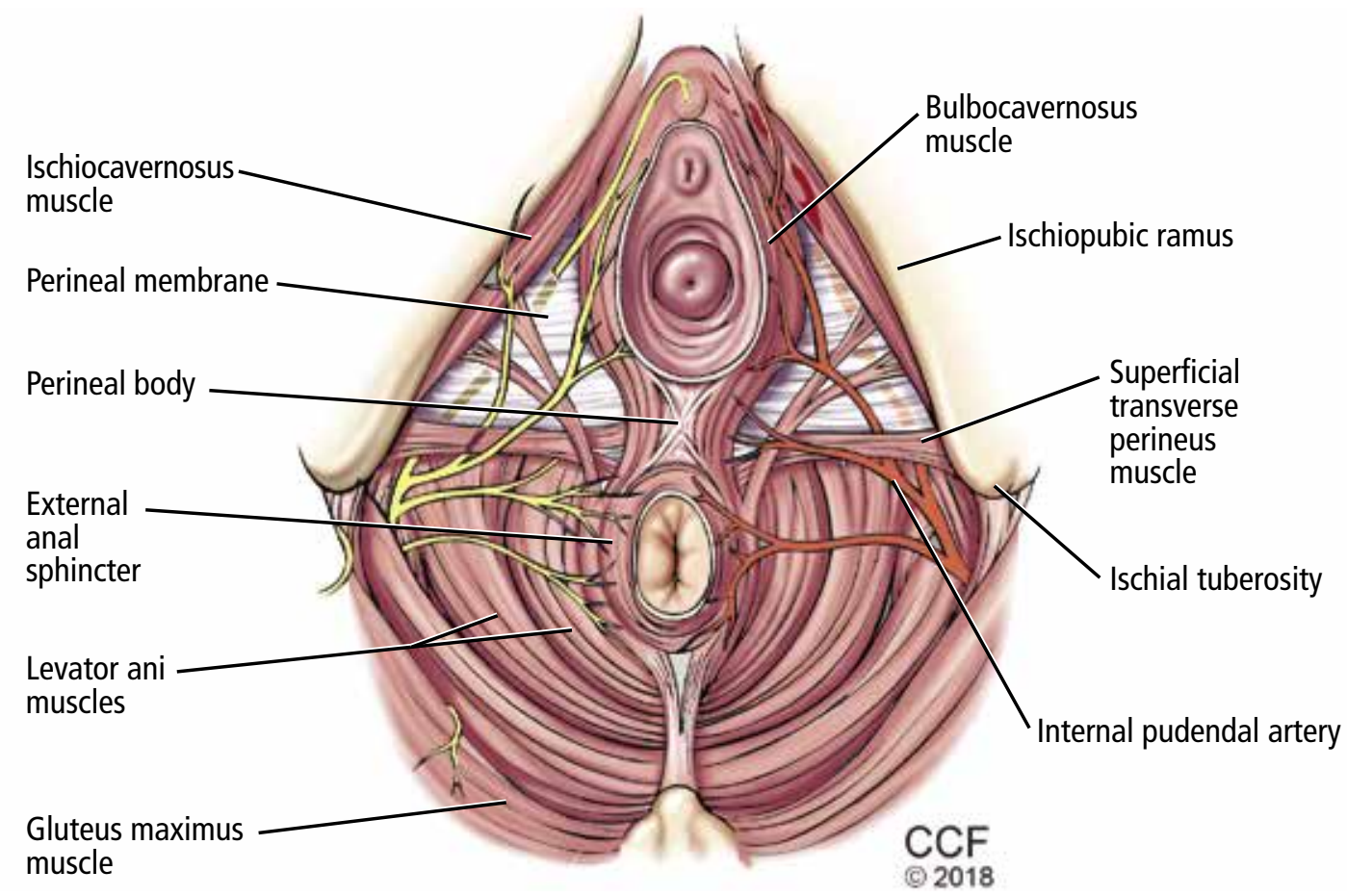

Figure 1. Pelvic floor anatomy. During the pelvic examination, the levator ani muscles should be directly palpated for tone and tenderness, and the pelvic floor should be evaluated with attention to any tenderness of the bladder and musculoskeletal structures.

acteristics of the pain as well as their medical and surgical history. Particular attention should be given to obtaining a complete gynecologic history, including pregnancy, delivery complications, dyspareunia, sexual assault, and trauma. A detailed review of systems should focus on the reproductive, gastroenterologic, musculoskeletal, urologic, and neuropsychiatric systems.

As with many pain syndromes, allowing the patient to "tell her story" helps to establish rapport and obtain a more complete assessment. Chronic pelvic pain has been associated with physical or sexual abuse as a child or adult, so is essential to foster the doctor-patient relationship and create a safe and open space for disclosure. ${ }^{3,6}$ It is important to screen women for safety at home as well as for satisfaction or dissatisfaction with their relationships with their spouse or partner and family.

\section{Physical examination}

The physical examination should be directed by the history but should always include abdominal and pelvic examinations. These should be conducted slowly and gently, assessing for areas of tenderness, masses, and other abnormalities. Clinicians should aim to pinpoint the exact anatomic locations of tenderness if possible. Ongoing dialogue facilitates this process by inquiring about pain at each point of the examination.

The pelvic examination should begin with visual inspection for redness, discharge, lesions, fissures, excoriations, and other abnormalities. A moistened cotton swab may be used to evaluate the vulva and vestibule for localized tenderness. The manual portion of the pelvic examination should begin with a single digit, noting any introital tenderness or spasm. Next, the levator ani muscles should be directly palpated for tone and tenderness. The pelvic floor should be evaluated with attention to tenderness of the bladder or musculoskeletal structures (Figure 1). A bimanual examination assessing uterine size and tenderness, nodularity, or a fixed, immobile uterus should be conducted.

\section{Diagnostic workup}

Because the differential diagnosis of chronic pelvic pain is broad, the diagnostic workup and testing should be based on findings of the

\section{Chronic pelvic pain has been associated with physical or sexual abuse}


history and physical examination. In general, extensive laboratory testing is of limited use for evaluating women with chronic pelvic pain. ${ }^{3,7}$

Urinalysis should be obtained for symptoms suggesting bladder involvement such as interstitial cystitis.

Pelvic ultrasonography can help identify pelvic masses palpated during the physical examination, but routine use of imaging is not recommended. ${ }^{3,7}$ If pelvic congestion syndrome is suspected, starting with pelvic ultrasonography is reasonable before incurring the risk or cost of computed tomography or magnetic resonance imaging. ${ }^{8}$

\section{GENERAL TREATMENT}

A holistic approach ensures that the treatment plan adequately addresses the physical, social, and psychological aspects of chronic pelvic pain. ${ }^{9}$ Treatments may include medication, pelvic physical therapy, and behavioral therapies (Table 2). As with other chronic pain syndromes commonly seen in primary care, the treatment plan may develop over multiple visits as the patient-provider relationship grows and as treatment response is evaluated.

Extensive

laboratory

testing and imaging are of limited value

\section{Medical therapy}

The main goals of medical therapy are to improve function and quality of life while minimizing adverse effects. General treatments include the following:

Analgesics. Nonsteroidal anti-inflammatory drugs and acetaminophen may provide pain relief, although there is weak evidence for their efficacy in treating chronic pelvic pain. ${ }^{9}$

Neuropathic agents. One of several available neuropathic agents commonly used in the treatment of chronic pain can be tried on patients who fail to respond to analgesics. Tricyclic antidepressants such as amitriptyline and imipramine decrease pain, reduce symptoms of depression, and improve sleep. ${ }^{10}$ The results of a small randomized controlled trial suggest that gabapentin is more effective than amitriptyline for reducing chronic pelvic pain. ${ }^{11,12}$ Published guidelines currently list both amitriptyline and gabapentin as first-line agents; nortriptyline and pregabalin are considered acceptable initial alternatives. ${ }^{9}$

Venlafaxine and duloxetine may help chronic pelvic pain, although specific evi- dence is lacking. Duloxetine may be an appropriate choice for women with chronic pelvic pain who also experience depression and urinary stress incontinence. ${ }^{9}$

Opioids. Opioid therapy should be considered only when all other reasonable therapies have failed..$^{10}$ Patients may develop tolerance or dependence, as well as opioid-induced adverse effects such as hyperalgesia., ${ }^{9,10}$ Guidelines recommend that primary care providers consult with a pain management specialist before prescribing opioids, and that patients be thoroughly counseled about the risks and side effects. ${ }^{9}$

Nerve block and neuromodulation. There is weak evidence for the use of these modalities for treating chronic pelvic pain. ${ }^{9}$ If used, they should be part of a broader treatment plan and should be performed by providers who specialize in management of chronic pain.

\section{DISEASE-SPECIFIC TREATMENT}

\section{Endometriosis: Hormonal therapy}

Pelvic pain that significantly fluctuates with the menstrual cycle may be caused by endometriosis, the most common gynecologic cause of chronic pelvic pain. Women with cyclic chronic pelvic pain should be empirically treated with hormonal therapy for at least 3 to 6 months before diagnostic laparoscopy is performed. ${ }^{13}$

Oral contraceptives, gonadotropin-releasing hormone $(\mathrm{GnRH})$ analogues, progestogens, and danazol have proven efficacy, although side-effect profiles differ significantly. In a comparative trial, patients treated with $\mathrm{GnRH}$ analogues had more improvement in pain scores compared with those treated with oral contraceptives, but they experienced a significant decrease in bone mineral density. ${ }^{11}$ The effects on bone mineral density associated with $\mathrm{GnRH}$ analogue therapy can be mitigated by "add-back" low-dose hormonal therapy (norethindrone, low-dose estrogen, or a combination of estrogen and progesterone), which may also provide symptomatic relief for associated hot flashes and vaginal symptoms. ${ }^{11}$

\section{Interstitial cystitis often accompanies endometriosis}

Recognizing that chronic pelvic pain may have more than one cause is important when developing a comprehensive care plan. In- 


\section{TABLE 2}

\section{Treatment options for chronic pelvic pain in primary care}

\section{Type of therapy}

\section{Medications}

Simple analgesics

Neuropathics

Hormonal therapies

Antispasmodics

Neuromodulators

Other

\section{Examples or description}

\section{Comments}

Acetaminophen, nonsteroidal antiinflammatory drugs (NSAIDs)

Amitriptyline, gabapentin

Oral contraceptives, gonadotropinreleasing hormone (GnRH) analogues, progestogens, danazol

Dicyclomine

Botulinum toxin $\mathrm{A}$

Pentosan polysulfate sodium
Avoid prolonged use of NSAIDs due to potential toxicities

Gabapentin may be more efficacious than amitriptyline

$\mathrm{GnRH}$ analogues are more effective for pain relief than oral contraceptive pills

May worsen constipation

Very effective for pelvic floor spasm

First-line therapy for interstitial cystitis

\section{Pelvic physical therapy}

Strengthening (up-training)

Relaxation (down-training)

Biofeedback

Vaginal dilators
Contracting the pelvic floor in isolation

Stretching, meditation, internal and external manual release of involved muscle groups

Surface electromyography in conjunction with strengthening and relaxation

Tool for stretching, pain-free vaginal insertion
Improves symptoms of pelvic heaviness and discomfort

Improves symptoms of chronic pelvic pain due to overactive pelvic muscles

Allows visual feedback on muscle control; useful for strengthening or relaxation and treatment of reflexive pelvic floor spasm

Restores flexibility and coordination of pelvic floor

\section{Psychosocial interventions}

Collaborative goal-setting

Identify what has improved patients current symptoms to develop goals
Very individualized and specific

Short-term goals

Assess progress during each visit and modify goals as needed

Establish a practice goal (number of practices per week) Allows for partner participation

Can be done with other relaxation techniques (eg, visualization, deep breathing)
Progressive muscle relaxation Systematic tensing and relaxing of each large muscle group progressing from toes to head terstitial cystitis coexists with endometriosis in up to $60 \%$ of patients. ${ }^{14}$ Initial treatment is pentosan polysulfate sodium, an oral treatment approved by the US Food and Drug Administration for interstitial cystitis that works by restoring the protective glycosaminoglycan layer in the bladder. ${ }^{14,15}$ Amitriptyline may also be used to treat interstitial cystitis-associated nocturia.

\section{Myofascial pain: Neuromuscular blockers}

According to a recent systematic review of therapies for chronic pelvic pain, patients 
with symptoms related to myofascial pain may benefit from neuromuscular blockade. ${ }^{12}$ One randomized controlled trial of the effectiveness of botulinum toxin $A$ vs saline for the treatment of chronic pelvic pain secondary to pelvic floor spasm found that after 6 months of observation, women who received botulinum toxin had significantly lower pain scores than those who received saline. ${ }^{12}$

\section{Pelvic congestion syndrome: Multiple options}

Pelvic congestion syndrome may be treated with hormonal, radiologic, or surgical therapy. ${ }^{16}$ A randomized controlled trial involving patients with chronic pelvic pain secondary to pelvic congestion demonstrated that treatment with medroxyprogesterone acetate or a GnRH agonist (goserelin) improved pelvic symptoms. ${ }^{17}$

A Cochrane review of nonsurgical interventions for chronic pelvic pain included women with a diagnosis of pelvic congestion syndrome or adhesions. It found that patients treated with medroxyprogesterone acetate were more likely to have $50 \%$ pain reduction lasting up to 9 months compared with patients

Recognizing

a possible multifactorial etiology to chronic pelvic pain is important taking placebo. ${ }^{12}$ In comparative studies, GnRH analogues were more effective in relieving pelvic pain than progestogen therapy.

Radiologic embolization therapy is as effective as hysterectomy for the relief of chronic pelvic pain related to pelvic congestion syndrome, and it can be performed in the outpatient setting.

\section{Irritable bowel syndrome: Try dietary changes}

Symptoms of chronic pelvic pain that are associated with changes in stool consistency and frequency suggest irritable bowel syndrome. Symptoms may improve with dietary changes and fiber supplementation. Antispasmodic agents are frequently used but their anticholinergic effects may worsen constipation. ${ }^{14}$

\section{PELVIC PHYSICAL THERAPY}

Pelvic physical therapy targets the musculoskeletal components of bowel, bladder, and sexual function to restore strength, flexibility, balance, and coordination to the pelvic floor and surrounding lumbopelvic muscles. $\mathrm{Pa}$ - tients with dyspareunia, pain with activity, or a significant musculoskeletal abnormality (eg, vaginismus or point tenderness on examination) are particularly good candidates for this therapy. It is done by a physical therapist with special training in techniques to manipulate the pelvic floor to address pelvic pain.

\section{Educating the patient}

Informing the patient before the initial physical therapy visit is essential for success. Referring clinicians should emphasize to patients that treatment response can help to guide further physician intervention. Patients should be counseled that pelvic physical therapy includes a pelvic examination and an expectation to participate in a home program. Although noticeable improvement takes time, encouragement provided by the entire team, including medical providers, can help a patient maintain her care plan.

Therapists typically see a patient once a week for 8 to 12 visits initially. Insurance usually covers pelvic physical therapy through the same policy as routine physical therapy.

During the initial evaluation, the patient receives an external and internal pelvic examination assessing muscle length, strength, and coordination of the back, hip, and internal pelvic floor. Internal evaluation can be done vaginally or rectally, with one gloved finger, without the need for speculum or stirrups. Biofeedback and surface electromyography (using either perianal or internal electrode placement) are used to evaluate muscle activity and to assist the patient in developing appropriate motor control during strengthening or relaxation. ${ }^{18}$

Up-training (or strengthening) aims to improve pelvic floor endurance. It can improve pelvic instability and symptoms of heaviness and discomfort from prolapse. Patients learn to appropriately utilize the pelvic floor in isolation. If a patient is too weak to contract on her own, neuromuscular electrical stimulation is used with an internal electrode to provide an assisted contraction.

Down-training (or relaxation) focuses on reducing tone in overactive pelvic muscles. It can improve symptoms of chronic pelvic pain, sexual pain, vulvodynia, and pudendal neuralgias. Patients are made aware of chron- 
ic holding patterns that lead to excess tone in the pelvic floor and learn how to release them through stretching, cardiovascular activity, meditation, and manual release of the involved muscle groups internally and externally. Internal musculature can be manipulated by a therapist in clinic or by the patient's trained partner; the patient can also reach necessary areas with a vaginal dilator.

Functional coordination of the pelvic floor is needed for comfortable vaginal penetration and defecation. Training with biofeedback improves a patient's ability to relax and open the pelvic floor. ${ }^{18}$ Vaginal dilators with surface electromyography are used to treat vaginismus to eliminate reflexive pelvic floor spasm during penetration. Perineal and vaginal compliance can be improved through manual release techniques with hands or vaginal dilators to restore normal mobility of tissues. This can reduce pain from postsurgical changes, postpartum sequelae, atrophic vaginal changes, shortened muscles from chronic holding, and adhesions.

\section{PSYCHOSOCIAL INTERVENTIONS}

Pelvic pain is not only a biomedical difficulty; psychosocial factors can contribute to and be affected by pelvic pain. Patients with pelvic pain often experience lower quality of life, higher rates of anxiety and depression, and increased stress compared with others. ${ }^{19,20}$ People with pain also have more relationship stress, and patients' partners often experience emotional distress, isolation, and feelings of powerlessness in the relationship. ${ }^{21}$

Psychosocial interventions, provided along with biomedical treatment, can help to reduce pain, anxiety, and depression and improve relational well-being. ${ }^{22,23}$ In addition to attending to pain-related symptoms, comprehensive care involves recognizing and treating coexisting anxiety, depression, stress, and relationship conflict. Interventions for these difficulties are many, and a comprehensive list of interventions is beyond the focus of this section. ${ }^{19}$

\section{Cognitive behavioral therapy}

Cognitive behavioral therapy is based on the idea that maladaptive cognitions can lead to problematic behaviors and emotional distress. ${ }^{24}$ Interventions are carried out by a provider with specialized training in its use (eg, therapist, pain psychologist, psychiatrist).

Meta-analyses of studies that investigated the efficacy of cognitive behavioral therapy for chronic pain found consistent small to medium improvement in pain-related symptoms. ${ }^{24}$ Studies that used cognitive behavioral therapy for pelvic pain found reduced overall pain severity and pain during intercourse, increased sexual satisfaction, enhanced sexual function, and less-exaggerated responses to pain. ${ }^{25-27}$

Although cognitive behavioral therapy and mindfulness-based interventions produce positive outcomes, research on these interventions typically includes treatment carried out over a span of weeks. Common barriers to such care include lack of patient motivation, financial limitations, transportation problems, and time constraints.

The following psychosocial interventions have been chosen because they can be delivered in a short amount of time and integrated into a patient's medical care by a medical or behavioral health provider. Because of the brevity and simplicity of these interventions, more patients with pelvic pain can receive psychosocial care as part of their usual medical encounters.

\section{Behavioral activation}

People experiencing depressive symptoms tend to isolate themselves and stop participating in activities they enjoy, including spending time with family and friends. Behavioral activation interventions that address such isolating behaviors have been shown to be effective in improving depressive symptoms. ${ }^{28-30}$

A simple, brief intervention can be administered during routine medical care, ${ }^{28}$ involving the following steps:

- Determine activities that the patient might implement that would decrease depressive symptoms. Questions such as, "When do you feel less depressed?" or "What brings you some happiness in your life?" can generate possible activities.

- Ask the patient to identify people in her life who have been supportive and with whom she could engage.

- Create with the patient a list of possible activities and social interactions that may enhance well-being.
Patients with dyspareunia are good candidates for pelvic physical therapy 


\section{TABLE 3}

\section{Collaborative goal-setting}

\begin{tabular}{ll} 
Individual & Couple \\
\hline My goal is: & Our goal is: \\
I will: & We will: \\
When: & When: \\
I will do this first: & We will do this first: \\
Challenges that may arise: & Challenges that may arise: \\
I will handle them by: & We will handle them by: \\
I will reward myself when: & We will reward ourselves when: \\
My reward will be: & Our reward will be:
\end{tabular}

- Make a schedule for participating in activities, possibly with rewards for completing them. Patients should be encouraged to follow the prescribed schedule of activities rather than make decisions based on mood or other factors.

\section{Relaxation strategies}

For all

interventions, setting goals is important
Relaxation can help patients reduce stress and anxiety, and can also help reduce pain. ${ }^{31-33}$

Diaphragmatic or "belly breathing" is a deep-breathing technique in which participants are asked to take in air through the nose and fully fill the lungs and lower belly. This technique allows the body to take in more oxygen, helping to lower blood pressure and slow the heartbeat. In addition to physiologic benefits, concentrating on deep breathing can help slow down or stop intrusive thoughts and distressing physical sensations. ${ }^{34}$

Progressive muscle relaxation involves the systematic tensing and relaxing of each large muscle group in the body. ${ }^{35}$ The goal is to eliminate physical and emotional stress through focusing on the sensations of tension and relaxation.

Scripts and audio and video resources for belly breathing and progressive muscle relaxation can be found on the Internet. The techniques can be taught during the medical appointment or offered as resources for home practice.

\section{Couple-based care}

Targeting couples is more effective for improving well-being than focusing solely on a patient's psychosocial difficulties, so each of the above interventions may be more effective if tailored to include the patient's partner. ${ }^{36}$ If the partner is with the patient during medical visits or is included in long-term psychosocial treatment, he or she can be directly involved in learning and practicing interventions with the patient. If the partner is not present, the patient can be asked to practice newly learned well-being-enhancing strategies with her partner outside the appointment time. Couples therapy can improve psychosocial well-being for both partners.

\section{Setting goals}

For all interventions, setting goals is important. Using collaborative goal-setting, patients and providers together should develop goals that specifically attend to reducing pain-related difficulties and comorbid anxiety, stress, and depression. ${ }^{22}$ For example, if a patient has found that belly breathing helps reduce anxiety, a goal may be for the patient to practice belly breathing with her partner 3 times per week for 10 minutes, until her next medical appointment in 1 month. After the patient and provider have codeveloped goals for improving psychosocial well-being, provider and patient can discuss progress made toward goals during each subsequent visit. Depending on progress, providers can highlight patient success and modify goals as needed. Table 3 provides an example outline of collaborative goal-setting.

\section{REFERENCES}

1. Zondervan KT, Yudkin PL, Vessey MP, et al. The community prevalence of chronic pelvic pain in women and associated illness behavior. Br J Gen Pract 2001; 51:541-547.

2. Mathias SD, Kuppermann M, Liberman RF, Lipschutz RC, Steege JF. Chronic pelvic pain: prevalence, health-related quality of life, and economic correlates. Obstet Gynecol 1996; 81:321-327.

3. Howard FM. Chronic pelvic pain. Obstet Gynecol 2003; 101:594-611.
4. AHRQ PCMH Resource Center. Transforming the organization and delivery of primary care. www.pcmh.ahrq.gov/. Accessed February 2, 2018.

5. Pryzbylkowski P, Ashburn MA. The pain medical home: a patientcentered medical home model of care for patients with chronic pain. Anesthesiol Clin 2015; 33:785-793.

6. Jamieson DJ, Steege JF. The association of sexual abuse with pelvic pain complaints in a primary care population. Am J Obstet Gynecol 1997: 177:1408-1412. 
7. Gambone JC, Mittman BS, Munro MG, Scialli AR, Winkel CA; Chronic Pelvic Pain/Endometriosis Working Group. Consensus statement for the management of chronic pelvic pain and endometriosis: proceedings of an expert-panel consensus process. Fertil Steril 2002; 78:961-972.

8. Ganeshan A, Upponi S, Hon LQ, Uthappa MC, Warakaulle DR, Uberoi R. Chronic pelvic pain due to pelvic congestion syndrome: the role of diagnostic and interventional radiology. Cardiovasc Intervent Radiol 2007; 30:1105-1111.

9. Engeler D, Baranowski AP, Elneil S, et al; European Association of Urology. Guidelines on chronic pelvic pain. http://uroweb.org/ wp-content/uploads/EAU-Guidelines-Chronic-Pelvic-Pain-2015.pdf. Accessed February 5, 2018.

10. Vercellini P, Vigano P, Somigliana E, Abbiati A, Barbara G, Fedele L. Medical, surgical and alternative treatments for chronic pelvic pain in women: a descriptive review. Gynecol Endocrinol 2009; 25:208221.

11. Rafique S, DeCherney AH. Medical management of endometriosis. Clin Obstet Gynecol 2017; 60:485-496.

12. Cheong YC, Smotra G, Williams AC. Non-surgical interventions for the management of chronic pelvic pain. Cochrane Database Syst Rev 2014; 3:CD008797.

13. Royal College of Obstetricians and Gynecologists. The initial management of chronic pelvic pain, Green-top guideline No.41. www. rcog.org.uk/globalassets/documents/guidelines/gtg_41.pdf. Accessed February 2, 2018.

14. Shin JH, Howard FM. Management of chronic pelvic pain. Curr Pain Headache Rep 2011; 15:377-385.

15. Nelson P, Apte G, Justiz R, Brismee JM, Dedrick G, Sizer PS. Chronic female pelvic pain-Part 2: differential diagnosis and management. Pain Pract 2012; 12:111-141.

16. Holloran-Schwartz MB. Surgical evaluation and treatment of the patient with chronic pelvic pain. Obstet Gynecol Clin North Am 2014; 41:357-369.

17. Soysal ME, Soysal S, Vicdan K, Ozer S. A randomized controlled trial of goserelin and medroxyprogesterone acetate in the treatment of pelvic congestion. Hum Reprod 2001; 16:931-939.

18. Arnouk A, De E, Rehfuss A, Cappadocia C, Dickson S, Lian F. Physical, complementary, and alternative medicine in the treatment of pelvic floor disorders. Curr Urol Rep 2017; 18:47.

19. Faccin F, Barbara G, Saita E, et al. Impact of endometriosis on quality of life and mental health: pelvic pain makes the difference. J Psychosom Obstet Gynaecol 2015; 36:135-141.

20. Naliboff BD, Stephens AJ, Afari N, et al; MAPP Research Network. Widespread psychosocial difficulties in men and women with urologic chronic pelvic pain syndromes: case-control findings from the multidisciplinary approach to the study of chronic pelvic pain research network. Urology 2015; 85:1319-1327.

21. West C, Usher K, Foster K, Stewart L. Chronic pain and the family: the experience of the partners of people living with chronic pain. J Clin Nurs 2012; 21:3352-3360.

22. Khatri P, Mays K. Brief interventions in primary care. www.integration.samhsa.gov/Brief_Intervention_in_PC,_pdf.pdf. Accessed February 2, 2018.
23. Roy-Byrne $\mathbf{P}$, Veitengruber JP, Bystritsky A, et al. Brief intervention for anxiety in primary care patients. J Am Board Fam Med 2009; 22:175-186.

24. Hofmann SG, Asnaani A, Vonk IJ, Sawyer AT, Fang A. The efficacy of cognitive behavioral therapy: a review of meta-analyses. Cognit Ther Res 2012; 36:427-440.

25. Masheb RM, Kerns RD, Lozano C, Minkin MJ, Richman S. A randomized clinical trial for women with vulvodynia: cognitive-behavioral therapy vs. supportive psychotherapy. Pain 2009; 141:31-40.

26. ter Kuile MM, Weijenborg PT. A cognitive-behavioral group program for women with vulvar vestibulitis syndrome (VVS): factors associated with treatment success. J Sex Marital Ther 2006; 32:199-213.

27. Bergeron S, Khalifé S, Glazer HI, Binik YM. Surgical and behavioral treatments for vestibulodynia: two-and-one-half year follow-up and predictors of outcome. Obstet Gynecol 2008; 111:159-166.

28. Cuijpers $P$, van Straten A, Warmerdam L. Behavioral activation treatments of depression: a meta-analysis. Clin Psychol Rev 2007; 27:318-326.

29. Mazzucchelli T, Kane R, Rees C. Behavioral activation treatments for depression in adults: a meta-analysis and review. Clin Psychol Sci Practice 2009; 16:383-411.

30. Riebe G, Fan MY, Unützer J, Vannoy S. Activity scheduling as a core component of effective care management for late-life depression. Int J Geriatr Psychiatry 2012; 27:1298-1304.

31. Chen YF, Huang XY, Chien CH, Cheng JF. The effectiveness of diaphragmatic breathing relaxation training for reducing anxiety. Perspect Psychiatr Care 2017; 53:329-336.

32. Klainin-Yobas P, Oo WN, Yew PYS, Lau Y. Effects of relaxation interventions on depression and anxiety among older adults: a systematic review. Aging Ment Health 2015; 19:1043-1055.

33. Finlay KA, Rogers J. Maximizing self-care through familiarity: the role of practice effects in enhancing music listening and progressive muscle relaxation for pain management. Psychology of Music 2015; 43:511-529.

34. Harvard Health Publications; Harvard Medical School. Relaxation techniques: breath control helps quell errant stress response. www. health.harvard.edu/mind-and-mood/relaxation-techniques-breathcontrol-helps-quell-errant-stress-response. Accessed February 2, 2018.

35. Bernstein DA, Borkovec TD. Progressive relaxation training: a manual for the helping professions. Champaign, IL: Research Press; 1973.

36. Whisman MA, Baucom DH. Intimate relationships and psychopathology. Clin Child Fam Psychol Rev 2012; 15:4-13.

ADDRESS: Rachel Bonnema, MD, MS, Division of General Internal Medicine, University of Nebraska Medical Center, 983331 Nebraska Medical Center, Omaha, NE 68198-3331; rbonnema@unmc.edu 